\title{
The Effect of the Yeast Probiotic Saccharomyces boulardii on Acute Diarrhea in Children
}

\author{
Abolfazl Mahyar ${ }^{1,{ }^{*}}$, Parviz Ayazi ${ }^{1}$, Haleh Pashaei ${ }^{1}$, banafsheh $\operatorname{arad}^{1}$, Sonia Oveisi ${ }^{1}$ and Shiva Esmaeili ${ }^{1}$ \\ ${ }^{1}$ Qazvin University of Medical Sciences, Qazvin, Iran \\ "Corresponding author: Qazvin University of Medical Sciences, Qazvin, Iran. Email: abolfazl473@yahoo.com
}

Received 2021 July 20; Revised 2021 October 10; Accepted 2021 October 16.

\begin{abstract}
Objectives: This study aimed to evaluate the effect of yeast probiotic Saccharomyces boulardii on the frequency and duration of acute diarrhea in children.

Methods: Sixty children were randomized to receive yeast probiotic S. boulardii in a daily dose of $250 \mathrm{mg}$ (trial group-30 patients) or placebo (control group-30 patients) for five days. The clinical and demographic characteristics of two groups were recorded before the intervention. After intervention, the two groups were compared in terms of frequency of diarrhea, duration of diarrhea, and hospital stay.

Results: The median (interquartile range) of frequency of diarrhea in the trial group was significantly lower than placebo group (4 [2] vs. $5[2.5])(\mathrm{P}=0.04)$. Also, the median (interquartile range) of duration of diarrhea in the trial group was significantly lower than placebo group (3 [2] vs. 4 [2.5]) ( $\mathrm{P}=0.03)$. There was no significant difference between groups for hospital stay $(\mathrm{P}=0.8)$.

Conclusions: The present study reveals that the use of yeast probiotic $S$. boulardii can reduce the frequency and duration of acute diarrhea. Therefore, we recommended S. boulardii administration to acute diarrhea in children.
\end{abstract}

Keywords: Acute Diarrhea, Saccharomyces boulardii, Children

\section{Background}

Acute diarrhea is one of the most common infectious diseases in children $(1,2)$. Based on reports of conducted studies, there are two billion cases of diarrhea in children worldwide per year, 1.9 million children younger than five years die as a result of diarrhea and $78 \%$ of mortality cases occur in developing countries (e.g., Africa and Southern Asia) (1-3). Diarrhea-related diseases are the cause of $9 \%$ of mortality in children and $18 \%$ of mortality in children younger than five years $(3,4)$. Every child younger than five years experiences diarrhea three times a year, and diarrhea is the second cause of death in this age group (3).

Acute diarrhea is characterized by semi-formed or watery stool occurring three times or more within 24 hours for a period of fewer than 14 days (3). Acute diarrhea is the most prevalent gastrointestinal disorder in children and is the main cause of dehydration (5). Diarrhea is frequently caused by viral or bacterial agents (4). Complications of acute diarrhea are dehydration, electrolyte disturbance, weight loss, chronic diarrhea, malnutrition, and mortality $(3,4)$. Given these complications, it would be necessary to take medical measures to prevent complications and reduce mortality. The main treatment of acute diarrhea is correction of dehydration depending on the severity of the dehydration (3-7).

Some reports have shown the effectiveness of adjunctive drugs in the treatment of acute diarrhea $(4,6,8-11)$. One of these drugs is Saccharomyces boulardii (8-12). There is a disagreement over the effect of $S$. boulardii in acute diarrhea (8-12). Some believe that the administration of this probiotic can reduce the frequency and duration of diarrhea (8-11). In contrast, Dalgic et al. believes that this probiotic has no effect on the treatment of acute diarrhea (12). Saccharomyces boulardii is a type of yeast probiotic from Saccharomyces cerevisiae species that has antiinflammatory and anti-bacterial effects (13-15). This study was conducted due to high prevalence of acute diarrhea in children and the conflict of opinions about the effectiveness of $S$. boulardii in treating acute diarrhea.

\section{Objectives}

The primary outcome of the study was the reduction of diarrhea frequency and diarrhea duration, and the secondary outcome was the reduction of hospital stay. 


\section{Methods}

This randomized clinical trial was conducted to evaluate the effect of yeast probiotic S. boulardii on the frequency and duration of acute diarrhea on sixty admitted children in Qazvin Children's Hospital in 2017 - 2018. This hospital is the only children's referral hospital in Qazvin province that is affiliated with Qazvin University of Medial Sciences (Qazvin, Iran). Children with the following criteria were enrolled in the study (inclusion criteria): (a) existence of acute diarrhea (watery or loose stool 3 times or more within 24 hours of a period of fewer than 14 days) (3,67), (b) mild to moderate dehydration (4), (c) normal stool examination and normal stool culture, (d) age between 2 - 5 years, and (e) no antibiotics or probiotic compounds were used during the last month. The children with bacterial diarrhea, chronic diarrhea, dysentery, malabsorptive disorders, associated diseases (e.g., diabetes, coeliac disease, dairy intolerance), severe dehydration (more than $9 \%$ dehydration), abdominal distension, ileus, and vomiting were excluded from the study.

\subsection{Sample Size}

Based on the previous study that was conducted on children affected with acute diarrhea (11). Our sample size (60 patients) was calculated according to: $\alpha=0.05, \beta=0.1$, $1-\alpha / 2=0.95,1-\beta=0.9$ (Power), $\sigma=2.1, \mu_{1}=5.5$ days (mean of diarrhea duration in control group), $\mu_{2}=3.4$ days (mean of diarrhea duration in trial group) and $\mathrm{d}=\left(\mu_{1}-\mu_{2}\right)=(5.5$ - 3.4 $)=2$.1. Consecutive sampling was continued until the desired sample size was reached.

\subsection{Study Design}

The eligible children were divided into two groups using balanced block randomization. In this method, primarily four blocks, including 15 randomized allocations were designed (AABB-BBAA-ABAB-BABA-BAAB-ABBA, ....). Next, numbers 1 - 4 were assigned 15 times according to the random numbers table. Then cards were prepared in the order of the above letters, and they were put in homochromatic envelopes. The referred patients entered the above groups in the mentioned order. The entry of the patients into the study was balanced and did not have the capability of being disclosed. Finally, the patients were divided into trial and control groups (each group30 patients). Group A (trial group) received $250 \mathrm{mg}$ /day of yeast probiotic $S$. boulardii for a period of five days, and group B (control group) received chickpea flour powder as placebo for five days after admission to the hospital. To make their consumption more convenient, the probiotic powder and chickpea flour powder were both solved into $10 \mathrm{~mL}$ of water and were given to the children. The groups were similar in terms of background and confounding variables such as gender, age, weight body mass index, etc. For prescribing S. boulardii, we used Yomogi cap (containing $250 \mathrm{mg}$ S. boulardii dried powder with the least amount of $10^{10} \mathrm{CFU} / \mathrm{g}$ produced by Ardeypharm GmbH, Herdecke, Germany, code no: 640920). The chickpea flour powder used in the present study was produced by Tarkhineh company with productivity license no: 81 and production license no: 16/19814, Tehran, Iran. The chickpea flour powder did not have any food coloring, flavors, or other additives. The placebo and S. boulardii powder were similar in terms of color, taste, and volume.

In this study, randomization and blinding were conducted by a person who was not part of the research project and was not engaged in the study. The patients and the parents of children were not informed of the treatment type used. The fluid therapy was similar in the two groups. In the first visit, the patients' information that was recorded is as follows: age, gender, height, weight, head circumference, degree of dehydration, diarrhea duration, and diarrhea frequency. Height, weight, and head circumference were measured according to the standard method (4). After the beginning of the treatment, the frequency of diarrhea and stool pattern were recorded daily for five days by pediatrics residents by reference to the mothers' reports.

Watery or loose stool for three times or more within 24 hours for a period less than 14 days was considered acute diarrhea $(3,6,7)$. Discontinuation of diarrhea was defined when the frequency of stool was less than three times/day and the stool consistency returned to normal pattern (11). The primary outcome of the study was the reduction of diarrhea frequency and diarrhea duration, and the secondary outcome was the reduction of hospital stay. Dehydration was defined into three types: minimal (less than $3 \%$, mild to moderate (3 - 9\%), and severe (> 10\%) (4). Fluid therapy was performed according to standard protocols (3, 4,6 ). All laboratory tests were performed in laboratory department of Qazvin Children's Hospital.

\subsection{Statistical Analysis}

The chi-square test was used for comparison of qualitative variables, and non-parametric test [Mann-Whitney $\mathrm{U}$ test; median (interquartile range [IQR])] were used for comparison of quantitative variables between groups. All statistical analyses were performed using SPSS for Windows 16.0 (SPSS Inc., Chicago, IL). P values $<0.05$ were considered statistically significant. 


\section{Results}

Among the 30 children in the trial group, 17 patients were male, while 13 patients were female. These values in the control group were 19 and 11 , respectively $(P=0.79)$. Before the intervention, there was no significant difference between the two groups in terms of age, weight, height, head circumference (up to three years of age), body mass index, diarrhea duration, and diarrhea frequency ( $P$ $>0.05$ )(Table1). After the intervention, the frequency of diarrhea and the duration of diarrhea in the group receiving S. boulardii were significantly less than those in the control group $(\mathrm{P}<0.05)$ (Table 2 ). However, no significant difference was observed in the groups in terms of the hospital stay $(\mathrm{P}=0.8)$ (Table 3$)$. Furthermore, there was a significant difference between groups in terms of weight gain before and after the intervention in each group $(\mathrm{P}=0.001)$ (Table 3). The patients did not have any side effects such as itch, urticaria, or rash.

\begin{tabular}{|c|c|c|c|}
\hline Variables & $\begin{array}{c}\text { Trial Group }(\mathbf{n}= \\
\text { 30) }\end{array}$ & $\begin{array}{c}\text { Control Group }(\mathbf{n}= \\
\text { 30) }\end{array}$ & $\mathbf{P}$ \\
\hline $\begin{array}{l}\text { Gender } \\
(\text { male/female })^{a}\end{array}$ & $17 / 13$ & $19 / 11$ & 0.79 \\
\hline $\operatorname{Age}(\mathrm{mo})^{\mathrm{b}}$ & $29(16.75)$ & $30.5(23)$ & 0.38 \\
\hline Weight $(\mathbf{k g})^{\mathbf{b}}$ & $13(3.38)$ & $13(4.88)$ & 0.66 \\
\hline Height $(\mathrm{cm})^{\mathrm{b}}$ & $88.25(16.13)$ & $89.75(15.75)$ & 0.29 \\
\hline $\begin{array}{l}\text { Head } \\
\text { circumference } \\
(\mathrm{cm})^{\mathrm{b}}\end{array}$ & $49(3)$ & $49.5(2.25)$ & 0.57 \\
\hline Temperature $\left({ }^{\circ} \mathrm{C}\right)^{\text {b }}$ & $37.2(0.6)$ & $37(0.55)$ & 0.29 \\
\hline Body mass index ${ }^{b}$ & $15.46(2.57)$ & $15.41(1.9)$ & 0.76 \\
\hline $\begin{array}{l}\text { Duration of } \\
\text { diarrhea (day) }\end{array}$ & $2(1)$ & $2(1)$ & 0.71 \\
\hline $\begin{array}{l}\text { Frequency of } \\
\text { diarrhea/day }\end{array}$ & $5(2.25)$ & $5(3)$ & 0.91 \\
\hline
\end{tabular}

\section{Discussion}

This study showed that $S$. boulardii was a yeast probiotic that led to a reduction in the frequency of diarrhea and the duration of diarrhea in children with acute diarrhea; however, it did not have any impact on the hospital stay. The studies investigating the impact of $S$. boulardii on acute diarrhea clash with each other $(8,10-12,16-20)$. The research of Das et al. on 60 children (aged five months-five years) with acute diarrhea caused by rotavirus revealed that the

\begin{tabular}{|c|c|c|c|}
\hline Variables & $\begin{array}{c}\text { Trial Group }(\mathbf{n}= \\
\text { 30) }\end{array}$ & $\begin{array}{c}\text { Control Group }(\mathbf{n}= \\
\text { 30) }\end{array}$ & $\mathbf{P}$ \\
\hline $\begin{array}{l}\text { Frequency of } \\
\text { diarrhea/day }{ }^{a}\end{array}$ & $4(2)$ & $5(2.5)$ & 0.04 \\
\hline $\begin{array}{l}\text { Duration of } \\
\text { diarrhea (day) }\end{array}$ & $3(2)$ & $4(2)$ & 0.03 \\
\hline$\underset{\text { a }}{\text { Hospital stay (day) }}$ & $4(3)$ & $4(2.2)$ & 0.8 \\
\hline
\end{tabular}

${ }^{\text {a }}$ Median (interquartile range [IQR]), Mann-Whitney U test.

Table 3. Comparison of Weight Gain Difference Before and After Intervention in Trial and Control Group

\begin{tabular}{lccc}
\hline Weight $(\mathbf{k g})$ & $\begin{array}{c}\text { Trial Group }(\mathbf{n}= \\
\mathbf{3 0})\end{array}$ & $\begin{array}{c}\text { Control Group }(\mathbf{n} \\
\mathbf{= 3 0})\end{array}$ & P \\
\hline $\begin{array}{l}\text { Weight gain } \\
\text { difference before } \\
\text { and after in each } \\
\text { group }\end{array}$ & $0.075(0.16)$ & $0(0.1)$ & 0.001 \\
\hline${ }^{\text {a }}$ Median (interquartile range $\left.[\mathrm{IQR}]\right)$, Mann-Whitney U test.
\end{tabular}

prescription of $S$. boulardii led to a reduction of diarrhea duration and hospital stay; however, it did not affect fever and vomiting. In this double-blind randomized controlled trial, 30 children received $250 \mathrm{mg}$ of $S$. boulardii, while another 30 received a placebo. The researchers pointed out that $S$. boulardii could be used to treat acute diarrhea caused by rotavirus (8).

Sharif et al. investigated 200 children (aged six months-six years) with watery acute diarrhea. They found that the prescription of $S$. boulardii significantly reduced diarrhea frequency and diarrhea duration. In their study, 100 children consumed $250 \mathrm{mg}$ of $\mathrm{S}$. boulardii per day plus ORS, while another group of 100 children received a placebo plus ORS (11). In a randomized double-blind controlled clinical trial, Grandy et al. investigated the condition of 64 infants (aged 1-23 months) with acute diarrhea caused by rotavirus. Their results showed that administration of boulardii significantly reduced diarrhea and fever compared to the findings in the control group. The treatment, however, did not affect the duration of hospitalization (16).

Corrêa et al. studied 186 children aged 6 - 48 months with acute diarrhea. They observed that the prescription of S. boulardii within 72 hours after acute diarrhea started could decrease diarrhea duration. Their study was a double-blind, randomized controlled clinical trial in which the intervention group received $200 \mathrm{mg}\left(4 \times 10^{9}\right.$ $\mathrm{CFU} / \mathrm{g}$ ) of $S$. boulardii twice a day for a period of five days; the control group, however, received a placebo (17). The findings of Feizizadeh et al. (10), and Villarruel et al. (19) stud- 
ies were similar to the findings of the studies mentioned above. In contrast, Dalgic et al. showed that the use of $S$. boulardii in the treatment of children with acute diarrhea caused by rotavirus had no significant impact on reducing fever, vomiting, diarrhea duration, and hospitalization duration, compared to the control group. In their study, six intervention groups that were consumed various compounds such as zinc, S. boulardii, and other types of probiotics, either mixed or pure, were compared to those of the control group. The study found that the prescription of $S$. boulardii mixed with zinc could significantly reduce diarrhea and the hospitalization duration. The researchers underscored mixed treatments (e.g., probiotics with zinc) in treating acute diarrhea caused by rotavirus (12).

Canani et al. studied 571 children with acute diarrhea who observed that the treatment of S. boulardii did not affect diarrhea frequency or diarrhea duration compared to the control group. The researchers stated that the treatment of acute diarrhea required the use of appropriate and effective probiotics (20). The results of the study are consistent with those of Sharifi et al. (11), Grandy et al. (16), and Corrêa et al. (17). The difference in the results of mentioned studies may be related to many factors such as sample size, age of studied patients, type of study, dose of $S$. boulardii used, and the cause of acute diarrhea.

Saccharomyces boulardii is tropical yeast that was first extracted from lychee and mangosteen fruits by the French scientist Henri Boulard in 1923 (13). Although early reports described distinct taxonomic, metabolic, and genetic properties for S. boulardii (synonym: S. cerevisiae var boulardii and S. cerevisiae Hansen CBS 5926); however, it was later found that this yeast was a strain of $S$. cerevisiae, sharing $>99 \%$ genomic relatedness (21). Saccharomyces boulardii reaches its maximum growth and activity at $37^{\circ} \mathrm{C}$, and its lyophilized form remains resistant to gastric acidity and bile (18).

Saccharomyces boulardii can prevent and treat acute diarrhea with different mechanisms such as prebiotic effects, toxin degradation effects, antimicrobial effects, physical barrier effects, effects on the microbiota, anti-secretory effects, trophic effects, and effect on the immune system (22). The prebiotic effects of this probiotic relate to the various compounds of the cell wall, such as chitin and mannoproteins glucans, which can prepare short-chain fatty acids as a suitable material for fermentation (22). This probiotic, with secreting 54-kDa protease, destroys toxins A-B released from clostridium difficile and prevents the binding of these toxins to the brush border receptors. This process could reduce the cytotoxic and enterotoxinic effects of clostridium difficile (23). The binding of mannose on the surface of $S$. boulardii through lectin receptors (adhesins) to pathogenic organisms can prevent the connec- tion of these organisms to the intestinal brush border (24). This probiotic repairs the intestinal barrier integrity by regulating E-cadherin recycling (22). The effect of this organism in preventing diarrhea caused by the use of antibiotics has been confirmed by reducing the intestinal microbial flora and quickly repairing intestinal bacteria after the use of antibiotics $(25,26)$.

It has been found that the $120-\mathrm{kDa}$ protease released from S. boulardii can affect the enterocytes of the small and large intestines and prevent adenylate cyclase stimulation; the results of this process are enterocytic cyclic adenosine monophosphate (cAMP) and chloride secretion (27). Saccharomyces boulardii affects the innate and adaptive immune system, activates the response of the inflammatory system, and prevents the secretion of fluids inside the lumen; as a result, it can prevent and treat diarrhea (22). The S. boulardii trophic effects have been confirmed, which affect intestinal enterocytes and increase disaccharide release and digestive enzymes such as alkaline phosphatase, protease, glucoamylase, sucrase, lactase, maltase, and $\mathrm{N}$-aminopeptidase (22).

Based on our study results and their compatibility with other studies in the literature, and given the fact that no side effects resulted from $S$. boulardii use; this probiotic can help the treatment of acute diarrhea. However, small sample size, lack of virological analysis, and the age of the subjects were the limitations of this study. The study can be replicated with a larger sample size, including participants with different age-groups, and could rely on instruments for virological analysis. We recommend that educational and treatment centers in each region set up protocols for treating diarrhea and provide them to physicians for better treatment of the disease. We recommend that $S$. boulardii should be added to all patients with acute diarrhea of viral origin (28).

\subsection{Conclusions}

The present study reveals that the use of yeast probiotic Saccharomyces boulardii can reduce the frequency and duration of acute diarrhea. Therefore, we recommended Saccharomyces boulardii administration to acute diarrhea in children.

\section{Acknowledgments}

Our thanks and best regards go to the Research Department of Qazvin University of Medical Sciences and parents of children for their corporations. 


\section{Footnotes}

Authors' Contribution: Abolfazl Mahyar: design of the work; Parviz Ayazi: drafting of the work; Haleh Pashaei: collection of data; Banafsheh Arad: drafting of the work and final approval of the work; Sonia Oveisi: interpretation of data; Shiva Esmaeily: analysis of data.

Clinical Trial Registration Code: IRCT2017090812897N3. Conflict of Interests: The authors declare that they have no conflict of interest.

Data Reproducibility: The data presented in this study are openly available in one of the repositories or will be available on request from the corresponding author by this journal representative at any time during submission or after publication. Otherwise, all consequences of possible withdrawal or future retraction will be with the corresponding author.

Ethical Approval: This project was approved by the Ethics Committee of Qazvin University of Medical Sciences (IR.QUMS.REC.1396.239).

Funding/Support: No funding/support was received. Informed Consent: The children were included in the study after their parents agreed and signed the informed consent form.

\section{References}

1. Walker CLF, Rudan I, Liu L, Nair H, Theodoratou E, Bhutta ZA, et al. Global burden of childhood pneumonia and diarrhoea. Lancet. 2013;381(9875):1405-16. eng. doi: 10.1016/s0140-6736(13)602226. [PubMed: 23582727]. [PubMed Central: PMC7159282].

2. Liu L, Oza S, Hogan D, Perin J, Rudan I, Lawn JE, et al. Global, regional, and national causes of child mortality in 2000-13, with projections to inform post-2015 priorities: an updated systematic analysis. Lancet. 2015;385(9966):430-40. eng. doi: 10.1016/s0140-6736(14)616986. [PubMed: 25280870].

3. Farthing M, Salam MA, Lindberg G, Dite P, Khalif I, SalazarLindo E, et al. Acute diarrhea in adults and children: a global perspective. J Clin Gastroenterol. 2013;47(1):12-20. eng. doi: 10.1097/MCG.0b013e31826df662. [PubMed: 23222211].

4. Behutta ZA. Acute gastroenteritis in children. In: Behrman R, Vaughan III V, editors. Nelson textbook of pediatrics. Phila: WB Saunders company; 2016. p. 1854-75.

5. Radlović N, Leković Z, Vuletić B, Radlović V, Simić D. Acute Diarrhea in Children. Srp Arh Celok Lek. 2015;143(11-12):755-62. eng. doi: 10.2298/sarh1512755r. [PubMed: 26946776].

6. World Health Organization. The treatment of diarrhoea: a manual for physicians and other senior health workers. World Health Organization; 2005. Contract No.: 9241593180.

7. World Health Organization. Diarrhoea: why children are still dying and what can be done. UNICF Press; 2009. Contract No.: 9241598417.

8. Das S, Gupta PK, Das RR. Efficacy and Safety of Saccharomyces boulardii in Acute Rotavirus Diarrhea: Double Blind Randomized Controlled Trial from a Developing Country. J Trop Pediatr. 2016;62(6):464-70. eng. doi: 10.1093/tropej/fmw032. [PubMed: 27283364].
9. Burande MA. Comparison of efficacy of Saccharomyces boulardii strain in the treatment of acute diarrhea in children: A prospective, single-blind, randomized controlled clinical trial. J Pharmacol Pharmacother. 2013;4(3):205-8. eng. doi: 10.4103/0976-500x.114603. [PubMed: 23960427]. [PubMed Central: PMC3746305].

10. Feizizadeh S, Salehi-Abargouei A, Akbari V. Efficacy and safety of Saccharomyces boulardii for acute diarrhea. Pediatrics. 2014;134(1):e17691. eng. doi: 10.1542/peds.2013-3950. [PubMed: 24958586].

11. Sharif MR, Kashani HH, Ardakani AT, Kheirkhah D, Tabatabaei F, Sharif A. The Effect of a Yeast Probiotic on Acute Diarrhea in Children. Probiotics Antimicrob Proteins. 2016;8(4):211-4. eng. doi: 10.1007/s12602-0169221-2. [PubMed: 27530282].

12. Dalgic N, Sancar M, Bayraktar B, Pullu M, Hasim O. Probiotic, zinc and lactose-free formula in children with rotavirus diarrhea: are they effective? Pediatr Int. 2011;53(5):677-82. eng. doi: 10.1111/j.1442200X.2011.03325.x. [PubMed: 21261786].

13. Malgoire JY, Bertout S, Renaud F, Bastide JM, Mallié M. Typing of Saccharomyces cerevisiae clinical strains by using microsatellite sequence polymorphism. J Clin Microbiol. 2005;43(3):1133-7. eng. doi: 10.1128/jcm.43.3.1133-1137.2005. [PubMed: 15750073]. [PubMed Central: PMC1081240].

14. McFarland LV. Systematic review and meta-analysis of Saccharomyces boulardii in adult patients. World J Gastroenterol. 2010;16(18):2202-22. eng. doi: 10.3748/wjg.v16.i18.2202. [PubMed: 20458757]. [PubMed Central: PMC2868213].

15. Dahan S, Dalmasso G, Imbert V, Peyron JF, Rampal P, Czerucka D. Saccharomyces boulardii interferes with enterohemorrhagic Escherichia coli-induced signaling pathways in T84 cells. Infect Immun. 2003;71(2):766-73. eng. doi: 10.1128/iai.71.2.766-773.2003. [PubMed: 12540556]. [PubMed Central: PMC145355].

16. Grandy G, Medina M, Soria R, Terán CG, Araya M. Probiotics in the treatment of acute rotavirus diarrhoea. A randomized, double-blind, controlled trial using two different probiotic preparations in Bolivian children. BMC Infect Dis. 2010;10:253. eng. doi:10.1186/1471-2334-10-253. [PubMed: 20735858]. [PubMed Central: PMC2940902].

17. Corrêa NB, Penna FJ, Lima FM, Nicoli JR, Filho LA. Treatment of acute diarrhea with Saccharomyces boulardii in infants. J Pediatr Gastroenterol Nutr. 2011;53(5):497-501. eng. doi: 10.1097/MPG.0b013e31822b7abo. [PubMed: 21734600].

18. Pothoulakis C. Review article: anti-inflammatory mechanisms of action of Saccharomyces boulardii. Aliment Pharmacol Ther. 2009;30(8):826-33. eng. doi: 10.1111/j.1365-2036.2009.04102.x. [PubMed: 19706150]. [PubMed Central: PMC2761627].

19. Villarruel G, Rubio DM, Lopez F, Cintioni J, Gurevech R, Romero G, et al. Saccharomyces boulardii in acute childhood diarrhoea: a randomized, placebo-controlled study. Acta Paediatr. 2007;96(4):538-41. eng. doi:10.1111/j.1651-2227.2007.00191.x. [PubMed:17306006].

20. Canani RB, Cirillo P, Terrin G, Cesarano L, Spagnuolo MI, De Vincenzo A, et al. Probiotics for treatment of acute diarrhoea in children: randomised clinical trial of five different preparations. Bmj. 2007;335(7615):340. eng. doi: 10.1136/bmj.39272.581736.55. [PubMed: 17690340]. [PubMed Central: PMC1949444].

21. Khatri I, Tomar R, Ganesan K, Prasad GS, Subramanian S. Complete genome sequence and comparative genomics of the probiotic yeast Saccharomyces boulardii. Sci Rep. 2017;7(1):371. eng. doi: 10.1038/s41598-017-00414-2. [PubMed: 28336969]. [PubMed Central: PMC5428479].

22. Moré MI, Vandenplas Y. Saccharomyces boulardii CNCM I-745 Improves Intestinal Enzyme Function: A Trophic Effects Review. Clin Med Insights Gastroenterol. 2018;11:1179552217752680. eng. doi: 10.1177/1179552217752679. [PubMed: 29449779]. [PubMed Central: PMC5808955].

23. Castagliuolo I, Riegler MF, Valenick L, LaMont JT, Pothoulakis C. Saccharomyces boulardii protease inhibits the effects of Clostridium difficile toxins $\mathrm{A}$ and $\mathrm{B}$ in human colonic mucosa. Infect Immun.1999;67(1):302-7. eng. doi: 10.1128/iai.67.1.302-307.1999. [PubMed: 9864230]. [PubMed Central: PMC96311]. 
24. Gedek BR. Adherence of Escherichia coli serogroup O 157 and the Salmonella typhimurium mutant DT 104 to the surface of Saccharomyces boulardii. Mycoses. 1999;42(4):261-4. eng. doi: 10.1046/j.14390507.1999.00449.x. [PubMed: 10424093].

25. Moré MI, Swidsinski A. Saccharomyces boulardii CNCM I-745 supports regeneration of the intestinal microbiota after diarrheic dysbiosis - a review. Clin Exp Gastroenterol. 2015;8:237-55. eng. doi: 10.2147/ceg.S85574. [PubMed: 26316791]. [PubMed Central: PMC4542552].

26. Swidsinski A, Loening-Baucke V, Schulz S, Manowsky J, Verstraelen H, Swidsinski S. Functional anatomy of the colonic bioreactor: Impact of antibiotics and Saccharomyces boulardii on bacterial composition in human fecal cylinders. Syst Appl Microbiol. 2016;39(1):67-75. eng. doi 10.1016/j.syapm.2015.11.002. [PubMed: 26723852].

27. Czerucka D, Rampal P. Effect of Saccharomyces boulardii on cAMP- and Ca2+-dependent Cl- secretion in T84 cells. Dig Dis Sci. 1999;44(11):2359-68. eng. doi: 10.1023/a:1026689628136. [PubMed: 10573387].

28. Siroosbakht S, Rezakhaniha B. A survey of pediatricians' views and practices regarding parents' request for prescribing antibiotics: A qualitative study. Arch Pediatr Infect Dis. 2019;7(3). 